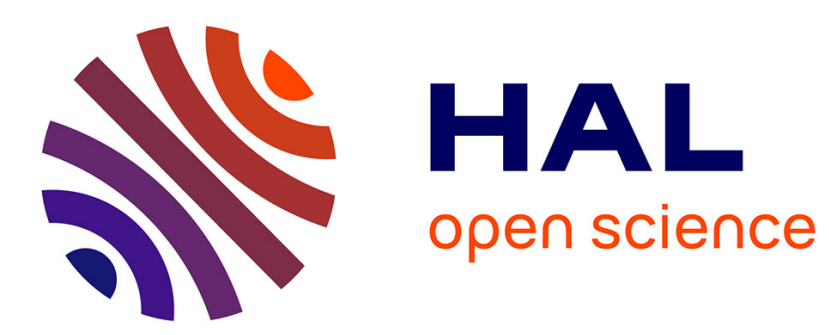

\title{
Numerical Resolution of the Cholesteric Unwinding Transition Problem
}

L. Gil

\section{To cite this version:}

L. Gil. Numerical Resolution of the Cholesteric Unwinding Transition Problem. Journal de Physique II, 1995, 5 (12), pp.1819-1833. 10.1051/jp2:1995106 . jpa-00248272

HAL Id: jpa-00248272

https://hal.science/jpa-00248272

Submitted on 1 Jan 1995

HAL is a multi-disciplinary open access archive for the deposit and dissemination of scientific research documents, whether they are published or not. The documents may come from teaching and research institutions in France or abroad, or from public or private research centers.
L'archive ouverte pluridisciplinaire HAL, est destinée au dépôt et à la diffusion de documents scientifiques de niveau recherche, publiés ou non, émanant des établissements d'enseignement et de recherche français ou étrangers, des laboratoires publics ou privés. 
Classification

Physics Abstracts

$6470 \mathrm{Md}-61.30 \mathrm{Cz}-11.30 \mathrm{Rd}$

\title{
Numerical Resolution of the Cholesteric Unwinding Transition Problem
}

\author{
L Gl
}

I N L N.(*), Unıversıté de Nıce Sophia Antipolis, 1361 Route des Lucıoles, F-06560 Valbonne, France

(Received 23 March 1995, revised 27 July 1995, accepted 14 September 1995)

\begin{abstract}
Some equilibrium configurations of the cholesterıc unwinding transition problem are numerically computed using the Landau-de Gennes expression of the liquid crystal free energy density The classical TIC and modulated TIC configurations are numerically obtained and the cholesteric finger structure is found to be in good agreement with the previous theoretical guesses. Moreover, we report the existence of new solutions, specifically associated with the local $\mathbf{n} \longrightarrow-\mathbf{n}$ symmetry, and which seem to be experimentally observed.
\end{abstract}

In the unwinding transition problem, a large-pitch cholesteric liquid crystal is sandwiched between two horizontal parallel glass plates, with homeotropic anchoring. When the distance $d$ between the two plates is small enough, the boundary conditions force all the molecules throughout the sample to be perpendicular to the plates. On the contrary, above a critical distance $d_{c}$, cholesteric winding takes place. In such a system, an ac high-frequency electric field is usually applied perpendicularly to the horizontal glass plates, in order to modify the molecular orientation through the dielectric anisotropy of the liquid crystal. The thermodynamical phase diagram is then very rich [1-3], displaying for example a homeotropic nematic phase, isolated cholesteric fingers (CF), fingerprint patterns [4], spherulitic bubble domains [5, 6], translationally invariant cholesteric configuration (TIC), and a modulated TIC phase [7]. The problem has already been the subject of a lot of experimental and theoretical studies. However to our knowledge, these various equilibrium configurations have never been exactly computed either analytically or numerically, and in general, even the theoretical computations of the critical lines of the phase space, brings into play an ansatz of the true solution and not the true solution itself.

In this paper, we report some equilibrium configurations obtained by the numerical resolution of the cholesteric unwinding transition problem. There are three main sections. The first one deals with the expression of the liquid crystal free energy density and the simplifying assumptions we have used. The second is devoted to the unusual numerical scheme. Finally the results are displayed and discussed in the last part.

(*) U.M R C N R S. 129.

(C) Les Editions de Physique 1995 
The thermodynamical phase diagrams of the cholesterıc unwinding transition problem can be analysed through the existence of a free energy density function $\mathcal{F}$, depending on the local variations of an unit vector $\mathbf{n}(\mathbf{r})$, which gives the direction of the preferred molecular orientation at each point $\mathbf{r}$ Certainly, the simplest derivation of the free energy density is due to Frank [8], who, on the base of symmetry arguments, has shown that $\mathcal{F}$ may be expressed as

$$
\mathcal{F}=\frac{K_{1}}{2}(\nabla \cdot \mathbf{n})^{2}+\frac{K_{2}}{2}\left(q_{0}+\mathbf{n} \cdot(\nabla \wedge \mathbf{n})\right)^{2}+\frac{K_{3}}{2}(\mathbf{n} \wedge(\nabla \wedge \mathbf{n}))^{2}+\mathrm{ST}
$$

where ST stands for surface terms, the $K_{\imath}$ are the anısotropic elastic constants of respectively splay, twist, and bend, and where $2 \pi / q_{0}$ is the cholesteric pitch. The symmetries involved are the invariance of $\mathcal{F}$ with respect to the three space rotations, and the $\mathbf{n} \stackrel{\text { global }}{\longrightarrow}-\mathbf{n}$ transformation. It is worth noting that this last transformation is global, meaning that the director $\mathbf{n}$ has to be changed in $\mathbf{- n}$ simultaneously in each point in space, and expresses the fact that the variations of $\mathcal{F}$ have to be independent from the way the vector $\mathbf{n}$ is defined with respect to the molecule axis. On the contrary, the fact that for most liquid crystals the two ends of a given molecule do not differ in an important way is carried on by the invariance of $\mathcal{F}$ with respect to the local transformation $\mathbf{n} \stackrel{\text { local }}{\longrightarrow}-\mathbf{n}$. As an illustration, the discontinuous or cutting lines observed in Figure 8a, only result from the use of a vector representation which does not preserve the local aspect of the $\mathbf{n} \stackrel{\text { local }}{\longrightarrow}-\mathbf{n}$ symmetry, but completely disappear in the corresponding nail representation of the director (Fig. 8b). Obviously, the Frank expression of the free energy density does not possess such a local invariance, and when one wants to rigorously describe some equilibrium configurations where this local aspect is suspected to play an important role, one has to deal with the Landau-de-Gennes expression of the free energy density [9]

$$
\left\{\begin{aligned}
\mathcal{F}= & A Q_{\imath \jmath} Q_{\jmath \imath}+\frac{C_{1}}{4}\left(Q_{\imath \jmath} Q_{\jmath \imath}\right)^{2}+\frac{B}{3} Q_{\imath \jmath} Q_{\jmath k} Q_{k \imath}+\frac{C_{2}}{4} Q_{\imath \jmath} Q_{\jmath k} Q_{k l} Q_{l \imath} \\
& +\frac{L_{1}}{2} \partial_{\imath} Q_{\jmath k} \partial_{\imath} Q_{\jmath}+\frac{L_{2}}{2} \partial_{\imath} Q_{\imath \jmath} \partial_{k} Q_{k \jmath}-2 L_{1} q_{0} \epsilon_{\imath \jmath} Q_{\imath l} \partial_{k} Q_{\jmath l} \\
Q_{\imath \jmath}= & \frac{S}{2}\left(3 n_{\imath} n_{\jmath}-\delta_{\imath \jmath}\right) \quad \text { with } \quad \operatorname{Tr}(Q)=0
\end{aligned}\right.
$$

Here, the spatial derivatives applying only onto the second order symmetric tensor $Q_{\imath \jmath}$, force the local $\mathbf{n} \stackrel{\text { iocal }}{\longrightarrow}-\mathbf{n}$ invariance, $S$ is an order parameter measuring how much the averaged molecular direction is well defined, $\delta_{\imath \jmath}=1$ for $\imath=\jmath$ and 0 otherwise, and $\epsilon_{\imath \jmath}$ is the LeviCevita anti-symmetric tensor of the third rank

Now for our purposes, some simplifying modifications have been done.

First, $S$ has been taken constant with respect to time and space. This assumption leads mainly to an incorrect description of the core of the topological defects and therefore to an incorrect estimation of the core contribution to the free energy. However the existence of topological defects $( \pm 1, \pm 1 / 2)$ is still allowed, and this because the defect stability is not related with the one of the core but with the stability of the regions asymptotically connected (which are here well described). The corresponding free energy density is then greatly simplified and is expressed as

$$
\left\{\begin{aligned}
\mathcal{F} & =F_{0}+\frac{L_{1}}{2} \partial_{\imath} Q_{\jmath k} \partial_{2} Q_{\jmath k}+\frac{L_{2}}{2} \partial_{\imath} Q_{\imath \jmath} \partial_{k} Q_{k \jmath}-2 L_{1} q_{0} \epsilon_{\imath \jmath} Q_{\imath \imath} \partial_{k} Q_{\jmath l} \\
Q_{\imath \jmath} & =\frac{1}{2}\left(3 n_{\imath} n_{\jmath}-\delta_{\imath \jmath}\right) \quad \text { with } \quad \operatorname{Tr}(Q)=0
\end{aligned}\right.
$$


where $F_{0}$ is constant. Now, it is very important to note the difference between the Frank free energy density expression and (1), and to understand the reason why we will use one instead of the other. In one hand, the Landau's expression (1) only contains two gradient terms ( $L_{1}$ and $\left.L_{2}\right)$ instead of three $\left(K_{1}, K_{2}\right.$ and $\left.K_{3}\right)$ for the Frank expression Therefore, expression (1) is, in this sense, an approximation of the Frank's expression where the usually good assumption $K_{1}=K_{3}$ has been made. But in the other hand, the Frank expression is unusable to deal with equilibrium configurations where the local aspect of the $\mathbf{n} \stackrel{\text { local }}{\longrightarrow}-\mathbf{n}$ symmetry is involved. Indeed, when $S$ is taken constant with respect to time and space, and when we restrict ourselves to regular equilibrium configurations with no cutting lines (i.e. to configurations where the local aspect of the $\mathbf{n} \stackrel{\text { local }}{\longrightarrow}-\mathbf{n}$ symmetry is not involved), 1 it is obvious to show that (1) can then be reduce to the Frank free energy expression (with $K_{1}=K_{3}$ ). Therefore the simplest idea to deal with some irregular configurations (as for example the $\pm 1 / 2$ topological defects), is first to assume the existence of a cutting line, and then to use the Frank's expression of the free energy to compute the director configuration far from this line. However this approach can reveal itself to be incomplete. Indeed, for example in the case of a pair of topological defects $+1 / 2$ and $-1 / 2$ connected by a cutting line $L$, the minimization of the Frank's free energy alone is not sufficient, because one has also to optimize the shape and the length of the curve $L$. In comparison, this optimization will be automatically performed with the use of expression (1), where $L$ is not a priori chosen, but, on the contrary, computed.

Second, it is convenient from a numerical point of view, to proceed to the following change of variables

$$
\left\{\begin{array} { r l } 
{ L _ { 1 } } & { = L _ { 1 } ^ { \prime } \times 1 0 ^ { - 1 2 } \mathrm { kg } \mathrm { m } \mathrm { s } ^ { - 2 } } \\
{ L _ { 2 } } & { = L _ { 2 } ^ { \prime } \times 1 0 ^ { - 1 2 } \mathrm { kg } \mathrm { s } \mathrm { s } ^ { - 2 } } \\
{ 4 L _ { 1 } q _ { 0 } } & { = u _ { 0 } ^ { \prime } \times 1 0 ^ { - 6 } \mathrm { kg } \mathrm { s } ^ { - 2 } }
\end{array} \quad \left\{\begin{array}{rl}
\partial_{\imath} & =\partial_{\imath}^{\prime} \times 10^{6} \mathrm{~m}^{-1} \\
\mathcal{F} & =\mathcal{F}^{\prime} \times 10^{-12} \mathrm{~kg} \mathrm{~m}^{-1} \mathrm{~s}^{-2}
\end{array}\right.\right.
$$

such that all the terms involved in the expression of the free energy density $\mathcal{F}^{\prime}$ (the prime will be dropped in the following) are now of order one.

Third, as in references [1-3]. we have assumed a strong homeotropic anchoring with the two glass plates such that surface energies have not to be considered.

Finally, we have restricted ourselves to the equilibrium configurations invariant by translation along at least one direction (defined as the $y$ direction) such that the problem can be investigated through a 2D $(x, z)$ analysis, $z$ being the vertical direction. For example, in the case of a cholesteric isolated finger, the finger axis has been assumed to be parallel to $y$, and therefore our simulations display the transverse structure of the finger.

From a numerical point of view, the minimization of the free energy $\mathcal{F}$ is performed by the numerical simulation of the Lyapunov's dynamics [10] associated with (1)

$$
\left\{\begin{aligned}
\frac{\partial n_{\imath}}{\partial t} & =-\frac{\delta \mathcal{F}}{\delta n_{\imath}} \\
& =-\frac{\delta \mathcal{F}}{\delta Q_{3 k}} \frac{\delta Q_{\jmath}}{\delta n_{\imath}} \\
n_{\imath} \in \Sigma &
\end{aligned}\right.
$$

where $\Sigma$ is the surface in the $\left\{n_{\imath}\right\}$ space defined by $n_{1}^{2}+n_{2}^{2}+n_{3}^{2}=1$, and $\gamma$ is a characteristic relaxation time, which by rescaling of the time $t$ will be taken equal to 1 . Hence defined, $\mathcal{F}$ is 
a decreasing function of time since

$$
\frac{\partial \mathcal{F}}{\partial t}=\frac{\delta \mathcal{F}}{\delta n_{\imath}} \frac{\partial n_{\imath}}{\partial t}=-\left(\frac{\delta \mathcal{F}}{\delta n_{\imath}}\right)^{2}<0
$$

which implies that the temporal evolution (2) leads necessary to a director configuration minimizing $\mathcal{F}$.

Now two important remarks are in order. First, although expressed with the component $n_{\imath}$ of the director, equation (2) really still possesses the local $\mathbf{n} \stackrel{\text { local }}{\longrightarrow}-\mathbf{n}$ symmetry. Second, from a numerical point of view, the expression (b) is much more convenient than (a) [11].

The previous equations have been integrated on a Connection Machine using a fourth order Runge-Kutta temporal scheme, the spatial derivatives being computed with a second order scheme. In order to take into account the constraint $n_{\imath} \in \Sigma$, the elementary displacement $\mathrm{d} n_{\imath}$ at each time step, is performed as the projection of $\left(\partial n_{\imath} / \partial t\right) \mathrm{d} t$ onto the surface $\Sigma$. The typical size of the grid point is $1024 \times 64$, with a space increment $\mathrm{d} r$ equal to 0.6 and a time increment $\mathrm{d} t$ to 0.01 . Checking simulations have being performed with either half $\mathrm{d} t$, or with $2048 \times 128$ mesh points, $(1 / 2) \mathrm{d} r$ and $(1 / 4) \mathrm{d} t$ without any significant changes. For $z=0$ and $z=63$, we have used the rigid boundary conditions $n_{3}= \pm 1$ in order to simulate a strong homeotropic anchoring, while for the $x$ axis, periodic boundary conditions have been employed. Finally, at each time step, we have also computed the total free energy of the system and rigorously checked its decrease with time as well as its convergence.

In a first step, we have paid particular attention to numerically obtain the usual cholesteric patterns reported in the literature, as the isolated finger [4], the translational invariant configuration or TIC and the modulated TIC [7] The ranges of parameters where to found these classical cholesteric configurations may be obtained analytically, by adapting the computational technique developed in references [1-3] for the Frank energy density, to those of Landau-de Gennes. Indeed, assuming a vertical dependence of the form

$$
\left(\begin{array}{l}
n_{1} \\
n_{2} \\
n_{3}
\end{array}\right)=\left(\begin{array}{c}
\sin (\phi) \cos (\theta) \\
\sin (\phi) \sin (\theta) \\
\cos (\phi)
\end{array}\right) \quad \text { with } \quad\left\{\begin{array}{l}
\phi=a \sin \left(\frac{\pi z}{d}\right) \\
\theta=q z
\end{array}\right.
$$

where $d$ is the distance between the two horizontal plates and $a$ the maximal azimuth deviation angle, we obtain that the value of $q$ which minimizes the free energy is

$$
q_{\mathrm{c}}=\frac{u_{0}}{2\left(2 L_{1}+L_{2}\right)}+\mathcal{O}\left(a^{2}\right)
$$

For this value, the free energy is expressed as

$$
\mathcal{F}(a)=\frac{c_{2}}{2} a^{2}+\frac{c_{4}}{24} a^{4}+\ldots \quad \text { with }\left\{\begin{array}{l}
c_{2}=-\frac{9}{8}\left(\frac{u_{0}^{2} d^{2}-4 \pi^{2}\left(2 L_{1}+L_{2}\right)^{2}}{d\left(2 L_{1}+L_{2}\right)}\right) \\
c_{4}=\frac{27}{4} \frac{u_{0}^{2} d}{\left(2 L_{1}+L_{2}\right)^{2}}\left(L_{1}-L_{2}\right)
\end{array}\right.
$$

Now some remarks are in order. First, the homeotroplc state, corresponding to $a=0$, is a stable solution provided that $c_{2}>0$, that is to say

$$
-u_{0_{\mathrm{c}}}<u_{0}<u_{0_{\mathrm{r}}}=2 \pi \frac{2 L_{1}+L_{2}}{d}
$$



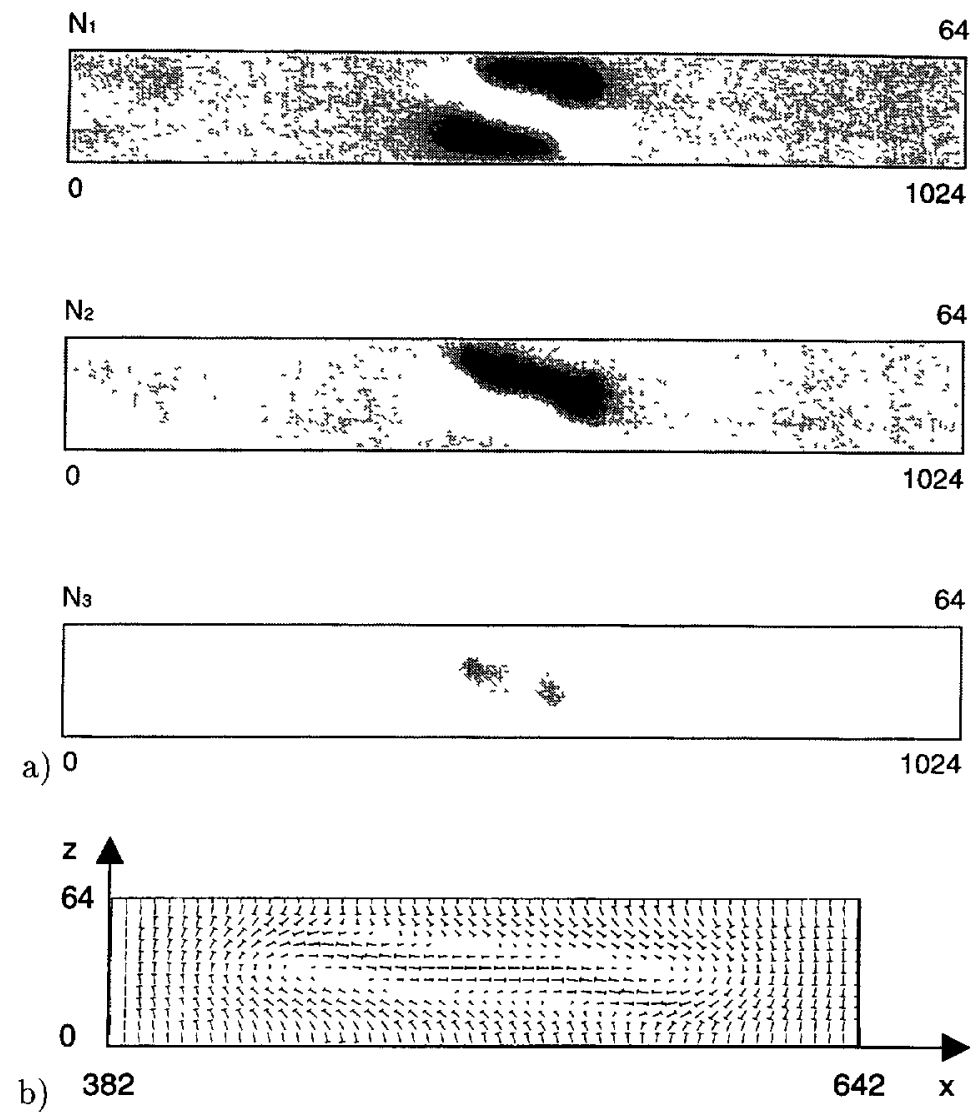

Fig. 1. - Numerical simulation of equation (2) with $1024 \times 64$ mesh points, $\mathrm{d} t=0.01, \mathrm{~d} r=06$, $L_{1}=1, L_{2}=6$ and $u_{0}=-10$, showing a Cholesteric Finger (CF) pattern. 1a) displays $N_{1}$ (resp $N_{2}$ and $N_{3}$ ) versus $(x, z)$, white corresponding to positive values of $N_{2}$, whlle black to negative ones. 1b) is the classical nails representation associated with $1 \mathrm{a}$ ).

Second, close to the cholesteric unwinding transition $u_{0} \simeq u_{0_{\mathrm{c}}}$, the value of $q$ minimizing $\mathcal{F}$ is $q_{\mathrm{c}}=\pi / d$, leading to a variation of $\theta$ between $z=0$ and $z=d$ independent of the value of $L_{1}, L_{2}$ and $d$, and equal to $\pi$. Third, a subcritical bifurcation may be observed for $c_{2}>0$ $\left(\left|u_{0}\right|<u_{0_{\mathrm{c}}}\right)$ and $c_{4}<0\left(L_{1}<L_{2}\right)$. Finally, it is worth noting that these analytical results are in perfect agreement with those, more general, dealing with the Frank's expression in presence of an electric field [1-3].

In our simulations, we have placed ourselves in the subcritical bifurcation range of parameters, in order to be able to observe localized patterns surrounded by the homeotropic phase (in most cases we have used $d=37.8, L_{1}=1$ and $L_{2}=6$, such that $\left|u_{0_{c}}\right|=1.33$ ). Figure 1 shows the transverse structure of a cholesteric finger. Then, changing the value of $u_{0}$ and $d$, we have been able to obtain the classical translational invariant configuration or TIC (Fig. 2), then a TIC making several winding through the sample thickness (Fig. 3), finally the same TIC with a spatial modulation along the $x$ axis (Fig. 4). These results are in good agreement with the topological models which have been deduced from the experimental observations [1-3,12], and in the case of Figures 2 and 3, with the analytical exact computations of reference [13]. In 
$N_{1}$ 64
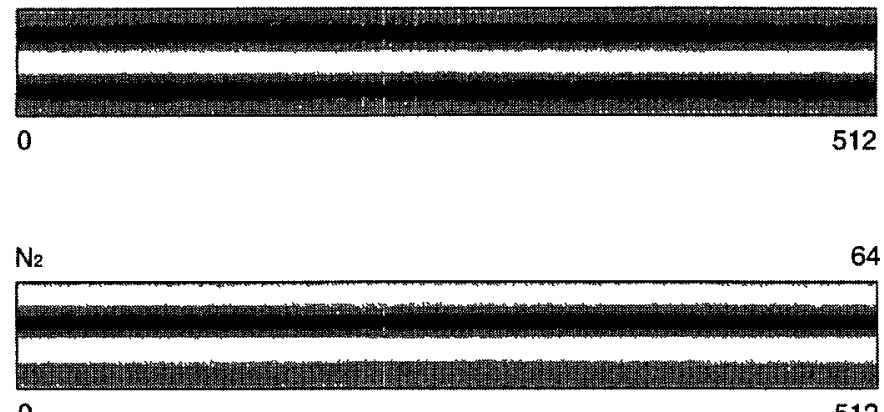

0
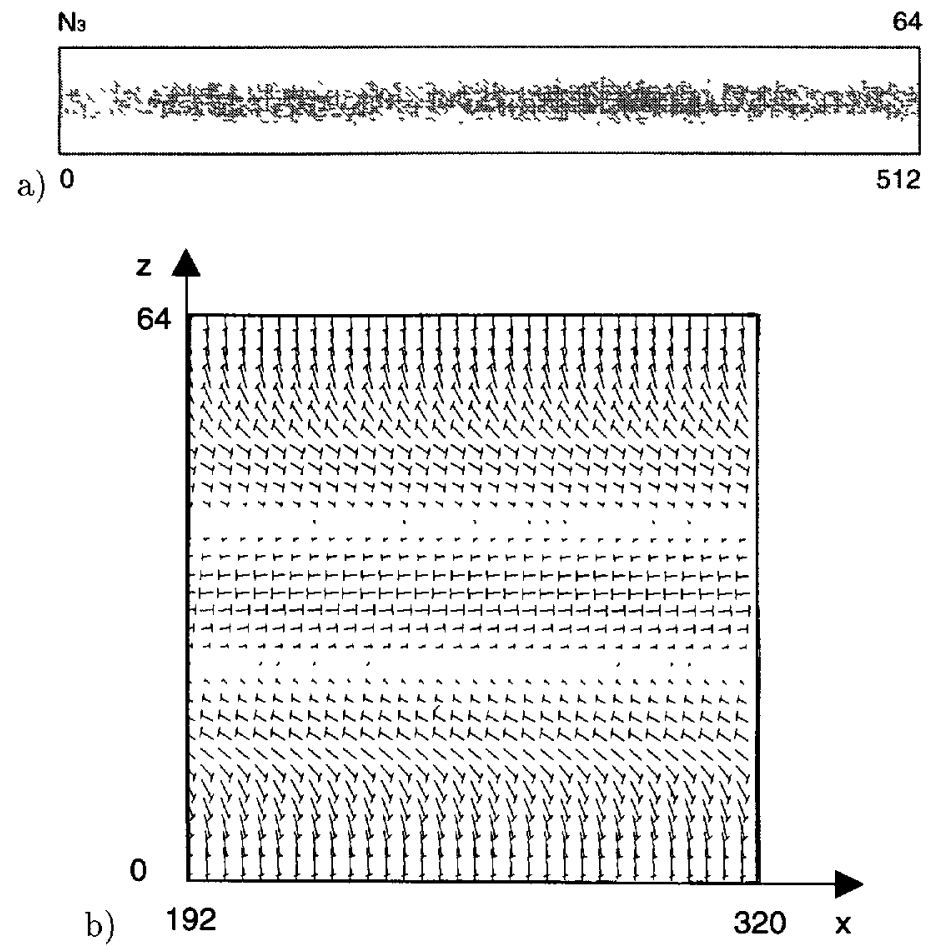

F1g. 2 - Numerical simulation of equation (2) with $512 \times 64$ mesh points, $\mathrm{d} t=0001, \mathrm{~d} r=0.3$, $L_{1}=1, L_{2}=6$ and $u_{0}=-3.0$, showing a TIC pattern, with only one wavelength along the $z$ axis. For this regime of parameter, the threshold value of $u_{0} 1 \mathrm{~s} u_{0_{\mathrm{c}}}=-2.659$. a) displays the components $N_{2}$ of the director versus $(x, z)$ and b) the corresponding nail representation.

fact, besides these quite classical equilibrium configurations, many other local minima of the free energy have been numerically obtained. As examples, Figures 5 and 6 display surprising cholesteric patterns which might be related to the experimental observations and theoretical models of references $[14,15]$.

Now we arrive to the new type of solutions, deeply associated with the local aspect of the $\mathbf{n} \stackrel{\text { local }}{\longrightarrow}-\mathbf{n}$ invariance. Let us first remind the difference between a topological wall and a 

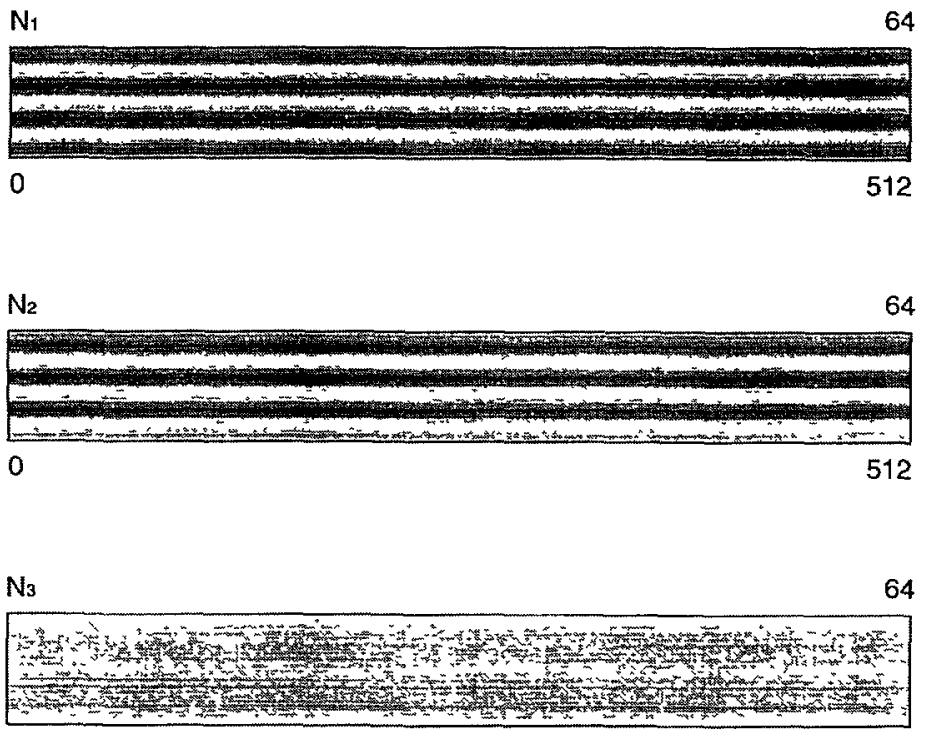

a) 0

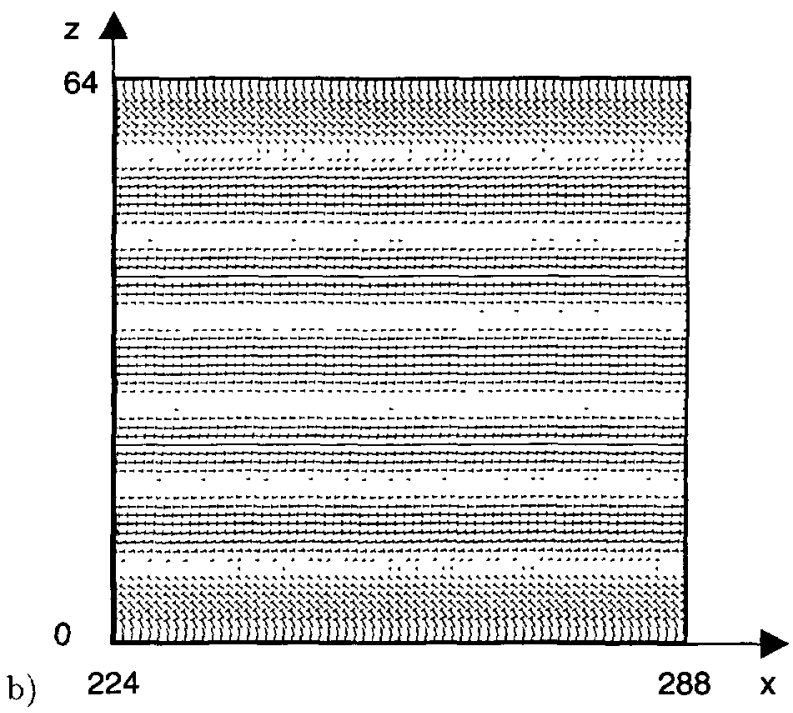

Fig 3. - Numerical simulation of equation (2) with $512 \times 64$ mesh points, $\mathrm{d} t=0.01, \mathrm{~d} r=0.6$, $L_{1}=1, L_{2}=6$ and $u_{0}=-30$, showing a TIC pattern, with three wavelengths along the $z$ axis. a) displays the components $N_{1}$ of the director versus $(x, z)$ and $\left.\mathrm{b}\right)$ the corresponding nail representation

cutting line. Walls are codimension one topological defects, connecting two solutions which can be exchanged one into the other by a discrete symmetry transformation. For example, because of the $\mathbf{n} \stackrel{\text { global }}{\longrightarrow}-\mathbf{n}$ invariance of equation (2), one can expect the exustence of walls separating the $(x, z)$ plane into two parts, one region being characterized by $\mathbf{n}=(0,0,+1)$, the other by $\mathbf{n}=(0,0,-1)$. For topological reasons, these walls are either closed lines, or open ones but with ends going to infinity, and because high gradients are usually forbidden due to 

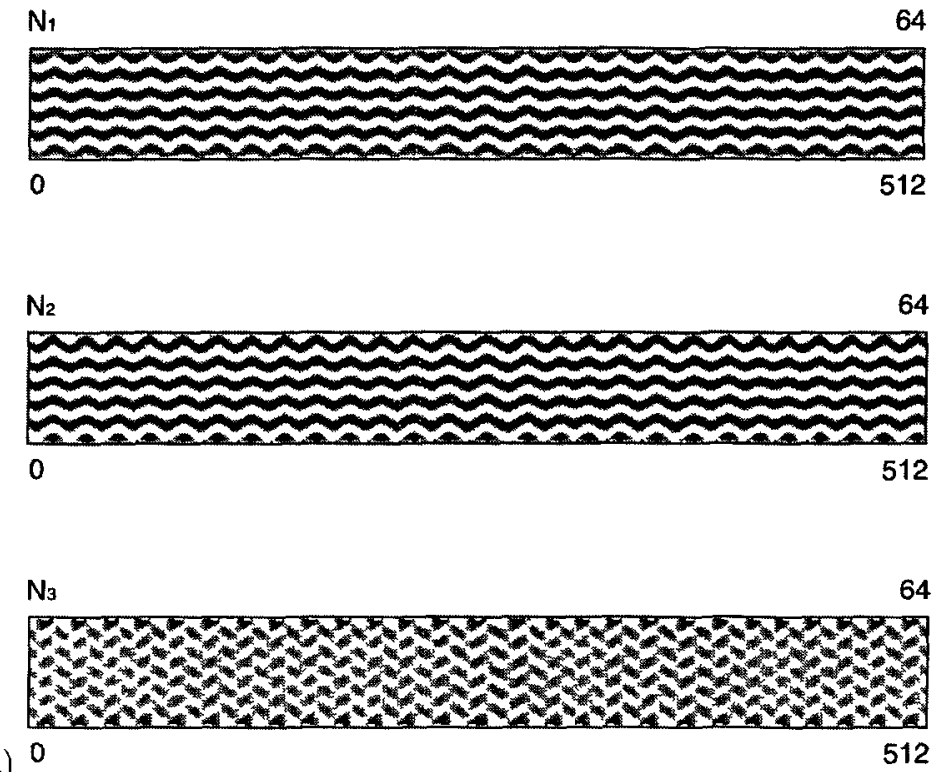

a) 0

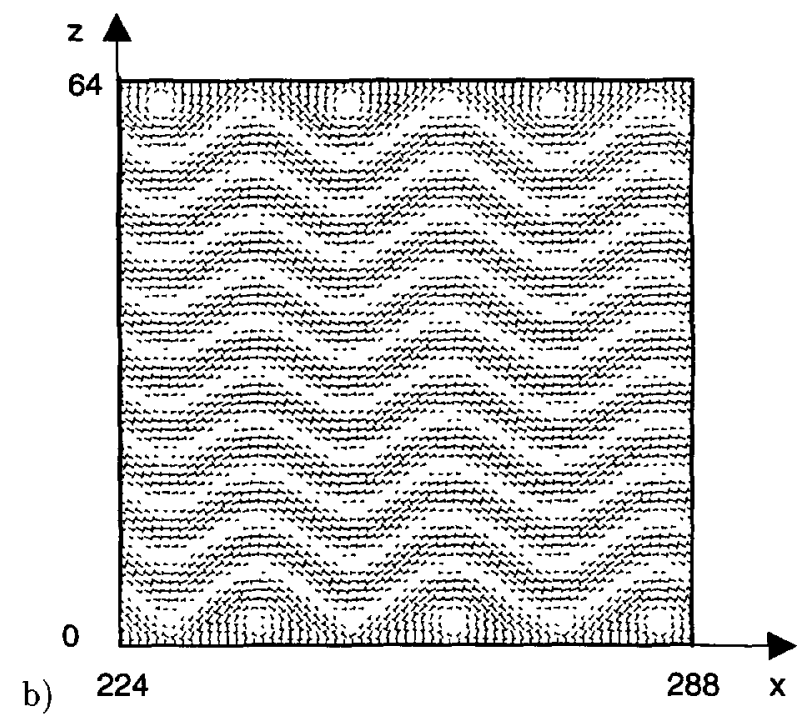

Fig. 4. - The same as for Figure 3, but now $u_{0}=-9.0$ a) displays the components $N_{2}$ of the director versus $(x, z)$ and $b$ ) the corresponding nail representation.

energetic considerations, the width of the defect core, defined as the distance needed for the director to make a $\pi$ rotation, has a non vanishing value. These topological defects have to be distinguished from the cutting lines, which are pure mathematical objects still separating the $(x, z)$ plane into a region with $\mathbf{n}=(0,0,+1)$ and a region with $\mathbf{n}=(0,0,-1)$, but characterized by a vanishing width. These lines are specifically associated with the local $\mathbf{n} \stackrel{\text { local }}{\longrightarrow}-\mathbf{n}$ invariance of the Landau-de Gennes free energy density expression, they cost no free energy, have no physical meaning and are not experimentally observable. 

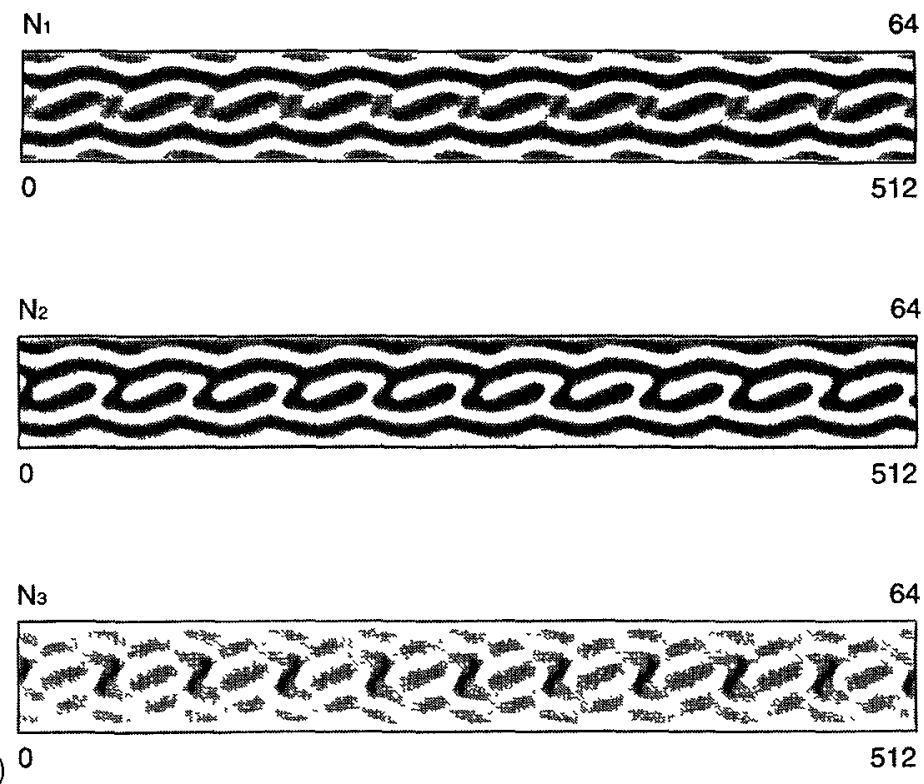

a) 0

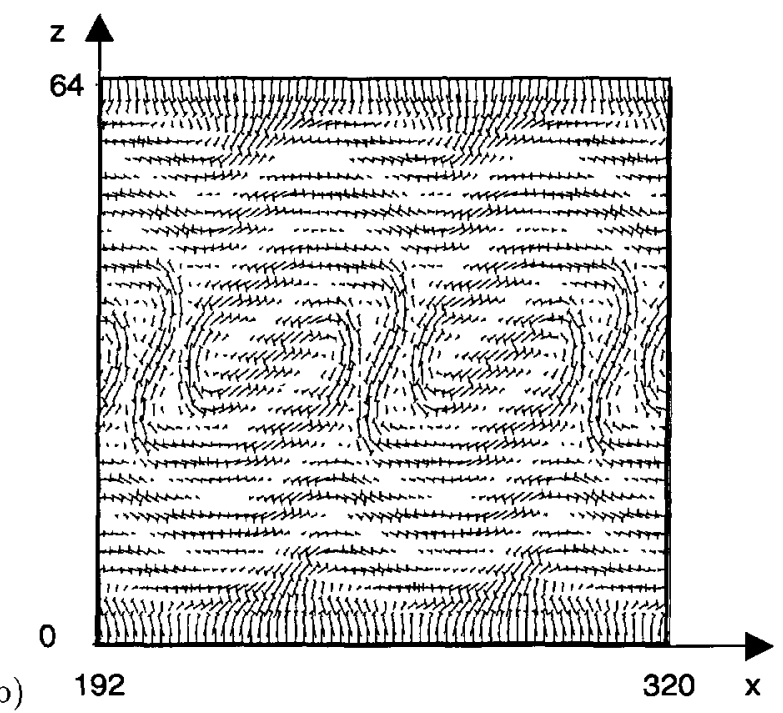

Fig. 5 - The same as for Figure 2, but now $u_{0}=-9.0$ a) displays the components $N_{2}$ of the director versus $(x, z)$ and $\mathrm{b})$ the corresponding nail representation.

Now, between these two limit situations, there exist intermediate configurations mixing the wall and the cutting line characteristics. We have performed the following numerical experiment As an initial condition, we start with a given cutting line, from which a continuous interval is suppressed and substituted with a wall pattern. This substitution is topologically possible, because the regions which are asymptotically connected, are the same for both the wall and the cutting line. Hence built, the resulting configuration still separates $a+\hat{z}$ to $a-\hat{z}$ region, and is compatible with the boundaries conditions used. There are of course some connecting troubles 


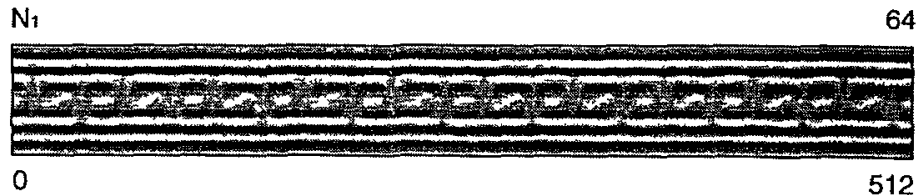

$\mathrm{N}_{2}$ 64

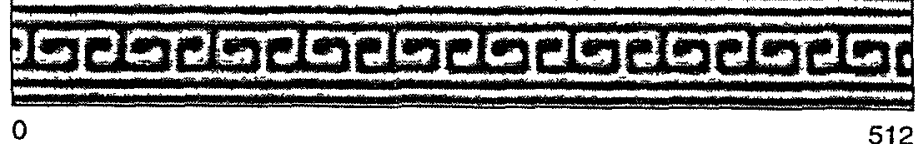

$\mathrm{N} 3$ 64

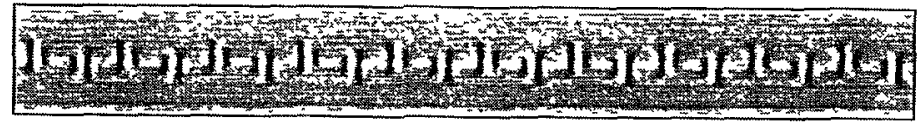

a)

0

512

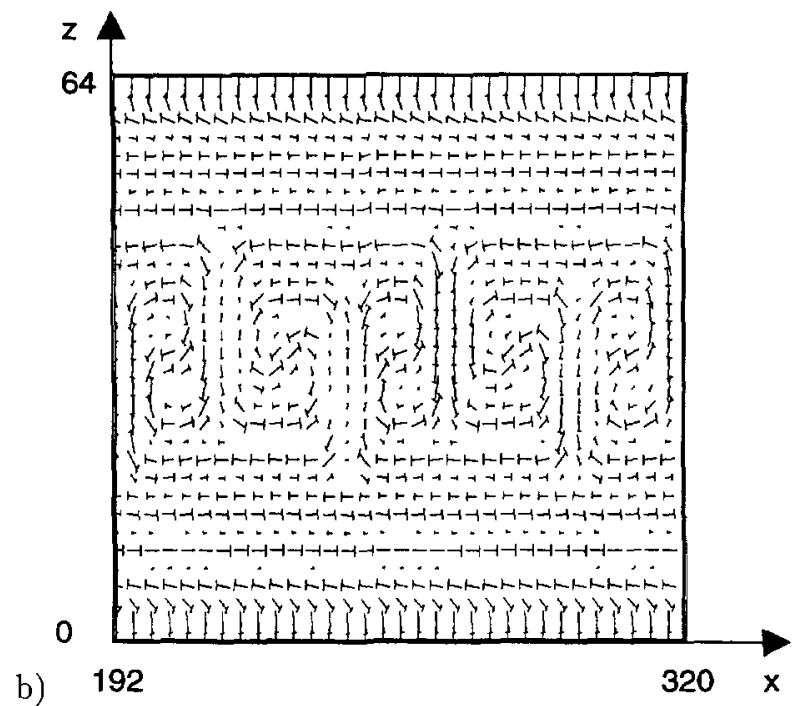

Fig. 6. - Numerical simulation of equation (2) with $512 \times 64$ mesh points, $\mathrm{d} t=001, d r=0.6$, $L_{1}=0.1, L_{2}=9$ and $u_{0}=-3.0$. This surprising pattern seems to be related to the experimental observations of references $[13,14]$. a) displays the components $N_{2}$ of the director versus $(x, z)$ and b) the corresponding nail representation.

at the junctions between the wall and the cutting line, but it has been found numerically that, starting from the initial conditions previously described, the system does converge to a free energy minimum. with a length and a width of the wall perfectly selected (this selection will never be possıble with the Frank description!). In our simulations, we have tried only two initial configurations. In the first one, the cutting line was initially vertical, in the second it was 

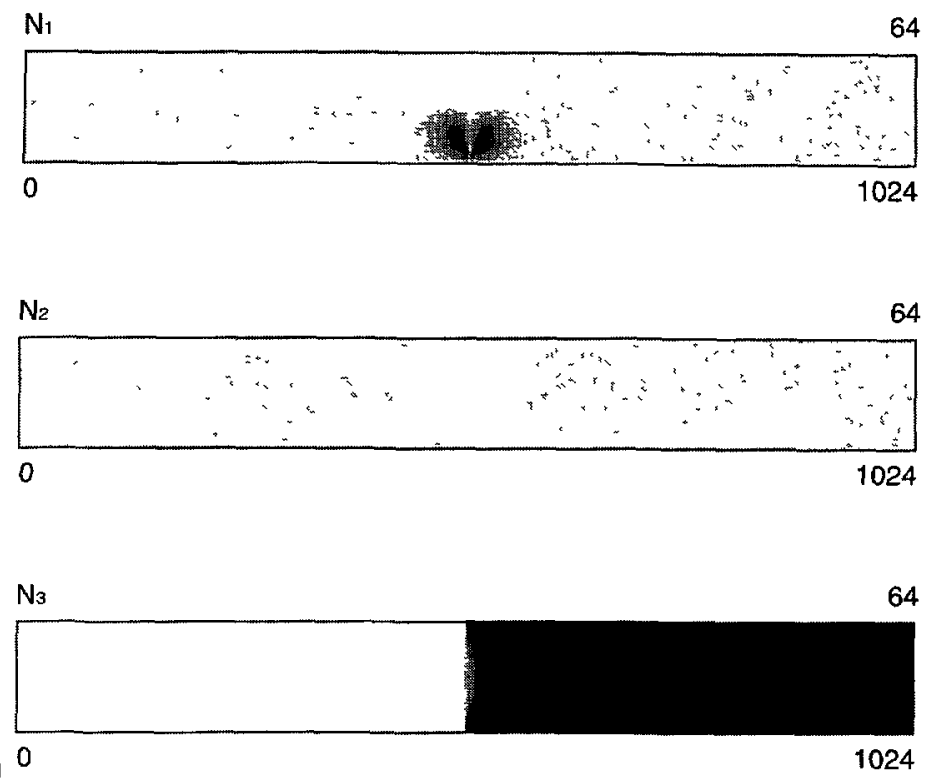

a) 0

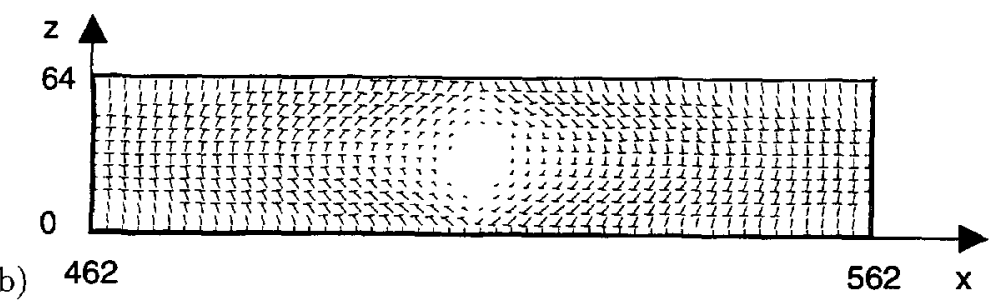

Fig. $T$ - Numerical simulation of equation (2) with the regime of parameter of Figure 1, displaying a Vertical Wall (VW) pattern a) displays the components $N_{\imath}$ of the director versus $(x, z)$ and b) the corresponding nail representation.

horizontal, initial configurations with an oblıque cutting line having never been investıgated. The respective converged patterns, which have been called vertical wall (VW) and horizontal wall (HW), are shown in Figures 7 and 8 . It can be checked that the cutting line only appear on the plots of $N_{\imath}$ versus $(x, z)$, but is not observable on the physical nail pictures.

Finally the diagram of Figure 9 displays the cost, in terms of the free energy, associated respectively with a cholesteric finger (CF), a vertical wall (VW) and a horizontal wall (HW) versus $u_{0}$. Note first that the respective free energy of the three patterns are almost the same, indicating that VW and $\mathrm{HW}$ patterns are almost as probable as the $\mathrm{CF}$, second, that there exists a transition from the VW to the $\mathrm{HW}$, bifurcation which may be understood as the tilting of the VW either on the left or on the right, and third that there exists a range of parameters for which the HW is the most stable pattern among the three configurations under consideration. 

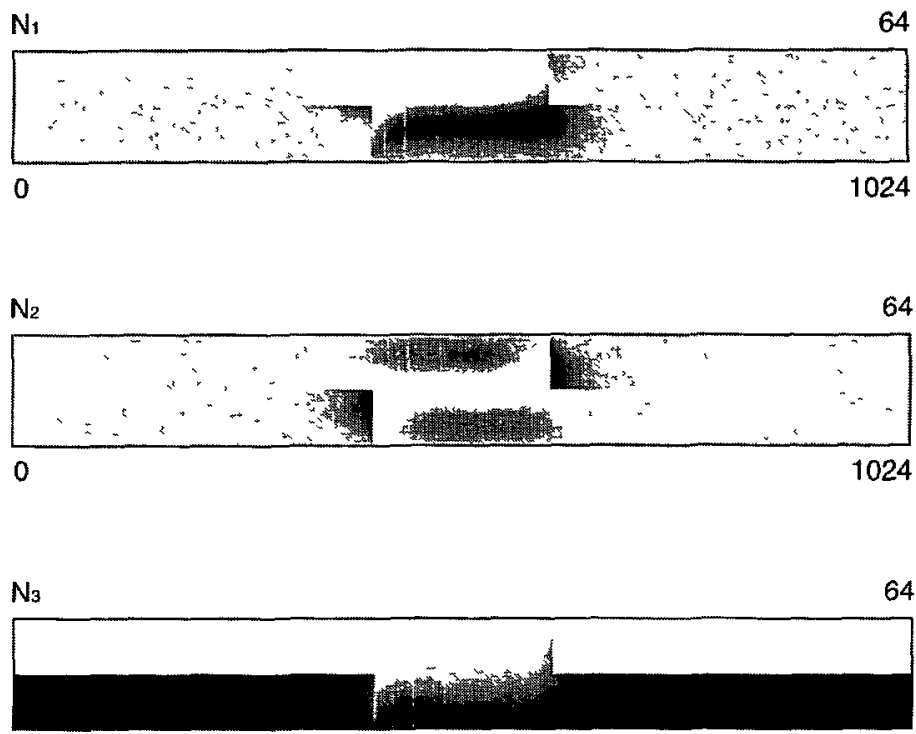

a) 0

1024

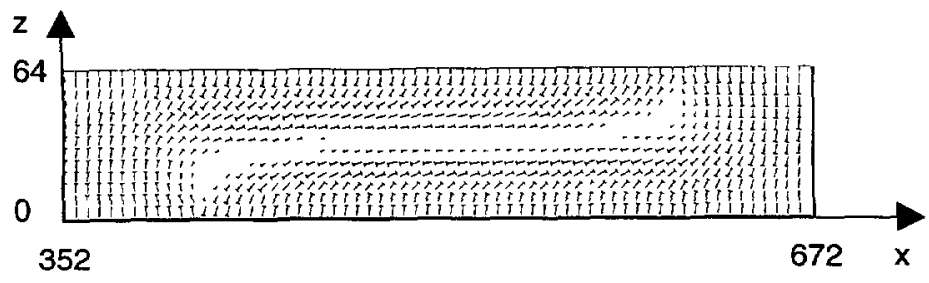

Fig. 8. - Numerical simulation of equation (2) with the regime of parameter of Figure 1, displaying a Horizontal Wall (HW) pattern a) display the components $N_{2}$ of the director versus $(x, z)$ and b) the corresponding nail representation

This tilting bifurcation seems to have been experimentally observed. In Figure 10a, by changing the focusing of the microscope from the lower to the upper glass plates, the thin structures pointed at by the arrow, are observed to be centered on the vertical axis. Although separating the space into two regions with a homeotropic phase, these thin patterns do not look like cholesteric finger (neither first nor second type [16]). Moreover, by changing the electrıc field frequency, these thin structures are observed to bifurcate and to give rise to thicker patterns (Fig. 10b). Again, by changing the focusing of the microscope, these thicker patterns are found to be tilted. As the tilting can be on the left or on the right, topological defects associated with the spontaneous breaking of a discrete symmetry and connecting left tilted patterns to right tilted are observed (Fig. 11).

Experimental works are in progress in order to absolutely identify these observed patterns with respectively our VW and HW solutions. 


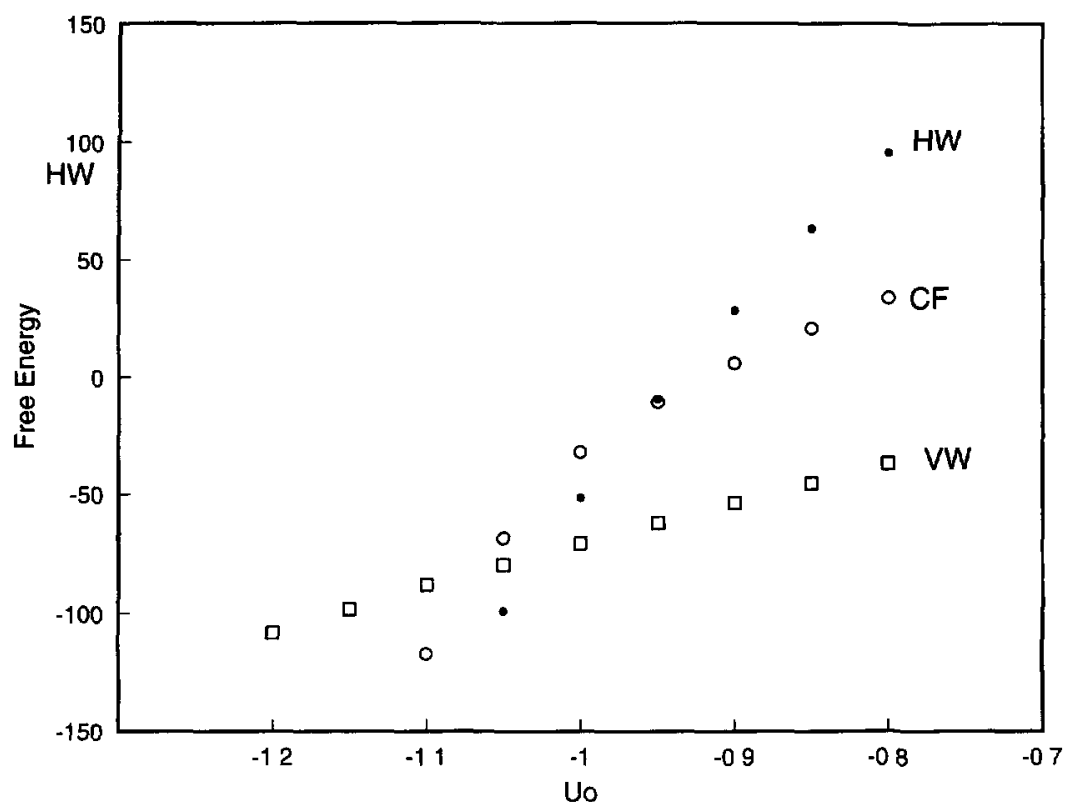

Fig. 9 - Numerical results with $1024 \times 64$ mesh points, $\mathrm{d} t=0.01, \mathrm{~d} r=0.6, L_{1}=1$ and $L_{2}=6$, showing the variations of the free energy $\mathcal{F}$ versus $u_{0}$, for a cholesteric finger $(\mathrm{CF})$, a vertical (VW) and a horizontal wall (HW).
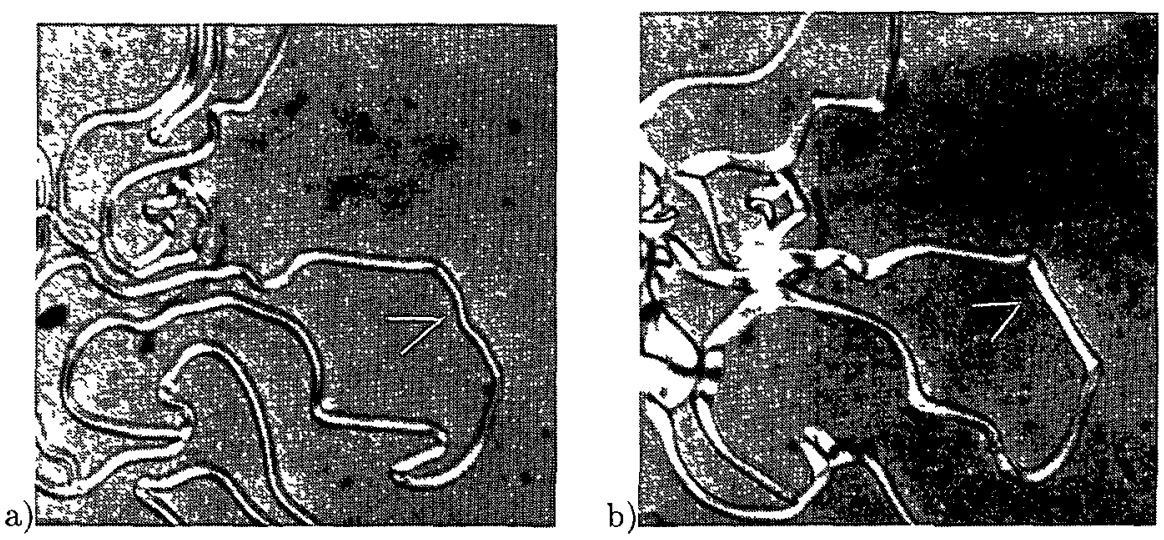

Fig 10. - Courtesy of J.M. Gilli Experimental observations of a cholesteric liquid crystal under a vertical ac electric field. In a), one can observe the thin patterns which we have identified with the VW structures. Then, decreasing the electric field frequency, an obvious thuckening of the structure may be observe in $b$ ) 


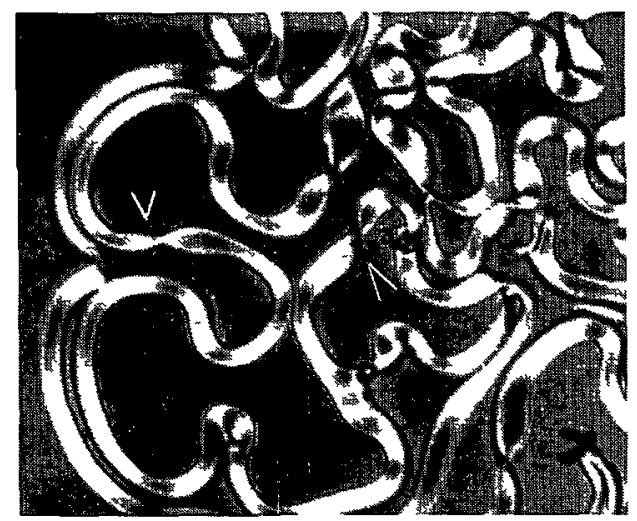

Fig 11. - Courtesy of J.M Gllh. Experimental observations of a cholesteric liquid crystal under a vertical ac electric field. The thickening bifurcation (Fig. 12) seems to be associated with a tilting of the Vertical Wall pattern either on the left or on the right (see text) Therefore, the transition is expected to give rise to topological defects connecting left and right tilted $\mathrm{HW}$ In this experimental picture, one can see the asymmetric aspect of the pattern as well as the expected topological defects (marked with an arrow)

\section{Acknowledgments}

We are very grateful to J L. Meunier and T. Frısch for their helpful discussions, and we especially thank J.M. Gilli to have allowed us to use his remarkable experimental results. We also acknowledge the I.N.R.I.A. Sophia-Antipolis France, where the numerical simulations have been performed. and Le Conseil Régional de la région Provence Côte d'Azur for its financial support.

\section{References}

[1] Lequeux F., Oswald P and Bechhoefer J., Phys Rev A 40 (1989) 3974.

[2] Ribıère P. and Oswald P., J. Phys France 51 (1990) 1703

[3] Ribière P, Pirkl S. and Oswald P., Phys. Rev A 44 (1991) 8198.

[4] Brehm M., Finkelmann H. and Stegemeyer H, Ber. Bunsenges Phys Chem. 78 (1974) 883.

[5] Haas W.E.L. and Adams J E, Appl Phys Lett 25 (1974) 263, 535

[6] Stieb A.E., J. Phys. France 4 (1980) 961

[7] Press M J. and Arrott A.S, J Phys France 37 (1976) 387.

[8] Frank F.C.. Discuss Faraday Soc. 25 (1958) 19

[9] de Gennes P G., Phys. Lett A 30 (1969) 454.

[10] Lyapunov A M., Problème Général de la stabilité du mouvement, Ann. Math. Studıes 17 (Princeton University Press, Princeton, NJ, 1947) 
[11] We illustrate this point with an example Let $\mathcal{F}\left(U^{2}\right)$ be defined as

$$
\begin{aligned}
\mathcal{F}\left(U^{2}\right) & =-U^{2}+\left(U^{2}\right)^{2}+\left(\frac{\partial U^{2}}{\partial x}\right)^{2} \\
& =-U^{2}+U^{4}+\left(2 U \frac{\partial U}{\partial x}\right)^{2}
\end{aligned}
$$

$\mathcal{F}$ being invariant under the local transformation $U \stackrel{\text { local }}{\longrightarrow}-U$ In presence of a cutting point, where $U$ is abruptly change into $-U$, expression (b) brings into play the indefined limit $(0 \times \infty)$. On the contrary, only regular limit are involved in expression (a) which is therefore much more convenient from a numerical point of view

[12] Frisch TY. Gil L and Gillı J M.. Phys Rev E 48 (1993) 4199, G1ll J.M and Gıl L, Lıq. Cryst. 17 (1994) 1.

[13] Fischer F, Z Naturforsch 31a (1976) 41

[14] Kohno T, Miike H. and Ebina Y., J Phys Soc. Jpn 44 (1978) 1678

[15] Hirata S, Matsuzaki I, Yanagita A and Tako T.. J. Phys Soc Jpn 50 (1981) 3862.

[16] Ribiere P, Oswald P. and Pırkl S, J Phys II France 4 (1994) 127 